\title{
APORTACIÓN AL CONOCIMIENTO DE LAS ALGAS EPICONTINENTALES DEL S.E. DE ESPAÑA. VIII: DESMIDIÁCEAS Y MESOTENIÁCEAS (DESMIDIACEAE, MESOTAENIACEAE, ZYGOPHYCEAE WIDDER 1960)
}

\author{
Marina ABOAL
}

\begin{abstract}
RESUMEN. En prospecciones algológicas estacionales de la cuenca del río Segura y puntuales en otras zonas del sudeste, se ha detectado una flora desmidial bastante rica para un área de arroyos calcáreos que consta de 48 táxones (21 pertenecen al género Cosmarium). De ellos 8 representan nueva cita para la flora algal española y 32 para la flora algal del sudeste ibérico. La media y los valores extremos de los principales factores físico-químicos del agua para cada especie se reúnen en tablas.
\end{abstract}

Palabras clave. Algas epicontinentales, Desmidiáceas, Mesoteniáceas, SE España, flora.

ABSTRACT. Some results of algological surveys carried out during last years in the river Segura Basin and other zones of Southeastern Spain are presented. Alkaline water streams are not a very usefull habitat for Desmidiales but 48 taxa were found ( 21 belongs to Cosmarium genera). Eight of them are cited for the first time for Spain and 32 are new for Southeastern Spain flora. Mean and extreme values of main physichal-chemical characteristics of water for each specie are summarized.

Key words. Freshwater algae, Desmidiaceae, Mesoteniaceae, Sutheastern Spain, flora.

\section{INTRODUCCIÓN}

En los últimos cinco años se realizaron prospecciones estacionales en los distintos sistemas acuáticos que constituyen la cuenca del río Segura (Aboal, 1987). Esta cuenca se caracteriza por su gran riqueza de ambientes; junto con sistemas temporales encontramos otros permanente, de agua dulce o salobre, de agua circulante o estancada (Mapa 1). 


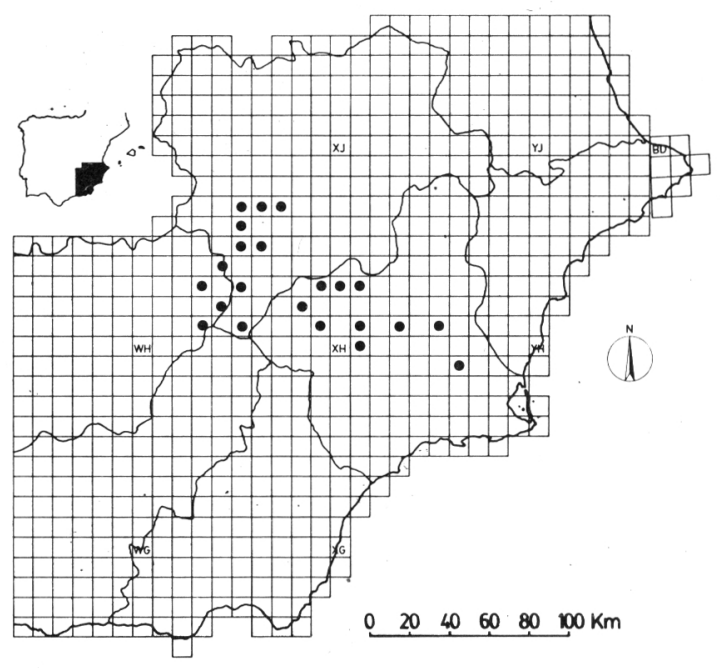

Mapa 1. Localización geográfica de la zona de estudio.

Los materiales sobre los que se asienta son calizos con algunas introgresiones de margas (del Neógeno o del Keuper) que permiten el desarrollo de arroyos salinos. Todos los sistemas acuáticos son alcalinos y con una dureza considerable (Vidal Abarca, 1985) lo que hace esperar una escasa diversidad de las Desmidiales en la zona debido a su preferencia por aguas finas, ligeramente ácidas.

De todas las estaciones consideradas (412) en el estudio global del río Segura (Aboal, 1987), en sólo 35 se ha podido constatar la presencia de estas conjugadas (Tabla I). Prácticamente todas ellas están ubicadas en los arroyos de la cabecera de la cuenca, con aguas puras, escaso caudal y escasa profundidad. Algunas, sin embargo, soportan ligeras muestras de eutrofia o una conductividad bastante elevada.

Con este estudio completamos el conocimiento hasta la fecha de las Zigofíceas (Zigophyceae) de la zona Sudeste. Previamente ya se conocía los datos de distribución y ecología de las Zignematáceas (Zignemataceae) (Aboal, 1988) y de algunas Desmidiáceas (Aboal y Llimona, 1984).

\section{MATERIALES Y MÉTODOS}

En la medida de lo posible el material se estudió en vivo. El fijador empleado fue una disolución de formaldehido al $4 \%$.

En algunos casos para facilitar la observación de las ornamentaciones de la pared se puede utilizar una solución alcohólica de hidróxido potásico.

Las monografías empleadas en la determinación específica han sido: Krieger \& Gerlof $(1962,1965,1969)$, Prescott et al. (1981) y West \& West (19041923). 


\section{RESULTADOS}

La casi totalidad de los táxones considerados se hallaron en relación con algas filamentosas, generalmente clorofíceas, formando parte del plocon, fijo a las rocas o flotando libremente y acumulándose en las riberas de los arroyos o en charcas ribereñas. Estas masas de filamentos constituyen un microambiente especialmente apto para el desarrollo de pequeños organismos vegetales y animales (Margalef, 1947).

En total se han reconocido 48 táxones de los que 8 representan nuevas citas para España (*) y 32 son citados por primera vez para el sudeste español (!) debido a la escasez de estudios previos.

Al comparar esta flora desmidial con la de áreas geográficas próximas y de análogas características de substrato sorprende comprobar la riqueza relativa de táxones del sudeste ibérico que puede llegar a duplicar el número conocido para Mallorca (Margalef, 1953) o Menorca (Margalef, 1952). Cabe destacar la diversificación de los géneros Closterium y Cosmarium con 13 y 21 táxones respectivamente.

El aspecto más destacable de esta flora desmidial es el claro predominio de las formas de pequeño tamaño y escasamente ornamentadas o lisas.

Para la mayoría de las especies se indican los valores de la media y la desviación típica de los principales factores físico-químicos del agua.

\section{F. MESOTAENIACEAE}

! Mesotaenium endlicherianum Nägeli (Fig. 1:8).

Atrapada entre los filamentos del plocon de los ríos (Tabla II). Insolación intensa.

Localidades: 1(III/84).- 2 (IX/85).

\section{! Cylindrocystis brevissonii Meneghini}

Vive entre los filamentos de otras algas en charcas poco profundas de las riberas de ríos (Tabla II). Las condiciones de iluminación son variables. Especie típica de superficies higropétricas (Margalef, 1955).

Localidades: 2 (IV/83).- 7 (VIII/83).

! Roya obtusa (Brébisson) W. West \& G.S. West var. montana W. West \& G.S. West.

Junto con muchas desmidiáceas vive entre clorofíceas filamentosas del plocon de charcas, a veces, muy someras de las riberas de los ríos (Tabla II) con fuerte insolación.

Localidad: 2 (XII/84).

! Gonatozygon monotaenium de Bary (Fig. 1:9)

Forma parte del plocon ribereño de cursos de agua alcalina dulce, con intensa insolación.

Localidades: 3 (IV/83, XII/84). 

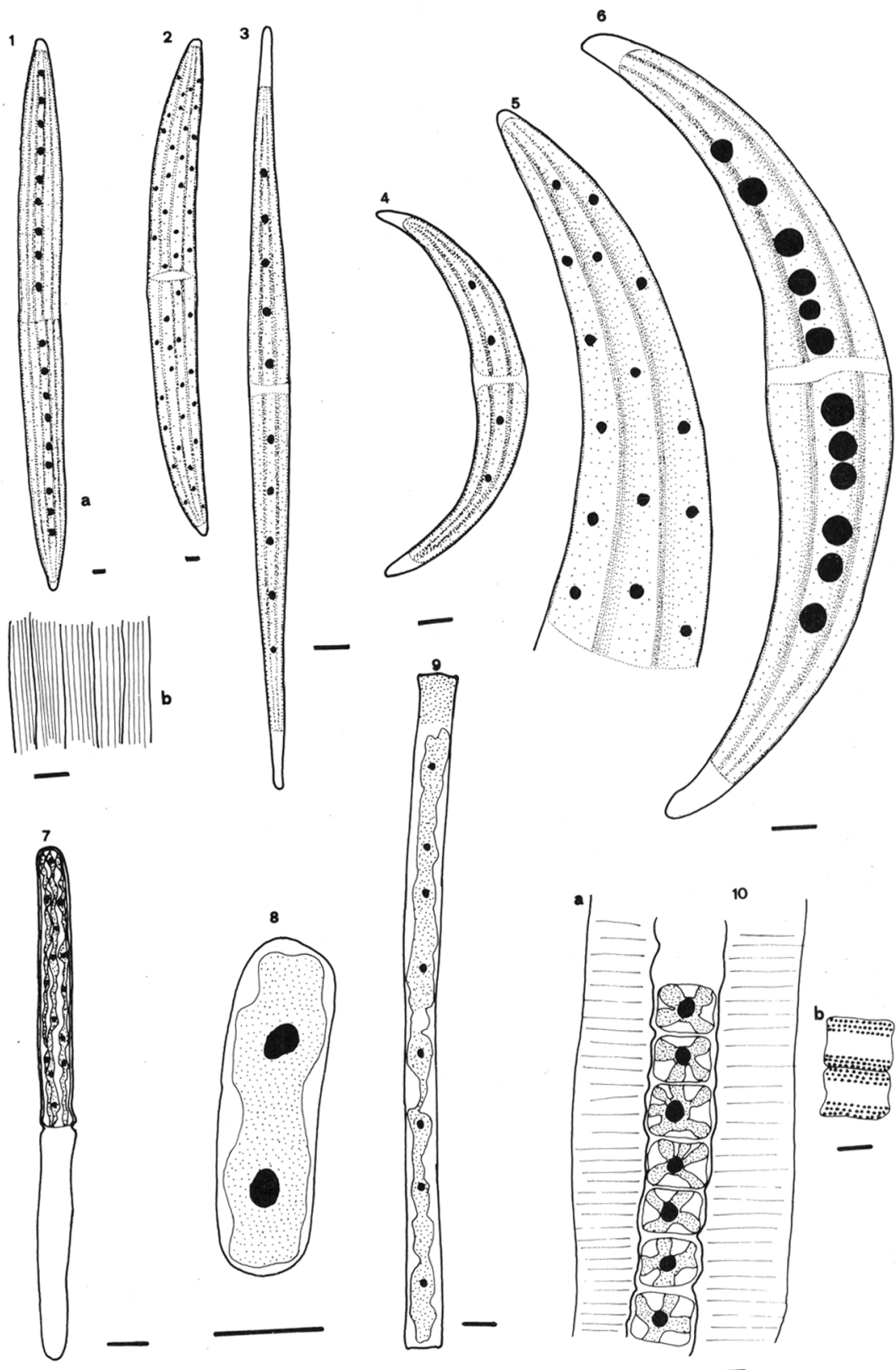

Figura 1: 1. Closterium acerosum.-2.C. lunula.- 3.C. strigosum var. elegans.- 4. C.parvulum.5.C.ehrenbergii.- 6.C.dianae.-7.Pleurotaenium trabecula.-8. Mesotaenium endlicherianum.9. Gonatozygon monotaenium.- 10. Hyalotheca dissiliens. (La escala representa $10 \mu \mathrm{m}$ ). 


\section{F. DESMIDIACEAE}

Closterium acerosum (Schranck) Ehrenberg (Fig. 1:1)

Entre los filamentos del plocon en cursos de agua. Soporta un cierto enriquecimiento del medio en materia orgánica (Tabla II). Especie típica de agua alcalina de lenta circulación (Margalef, 1955).

Localidades: 12 (VI/83).- 2 (III/84).- 11 (V/82).- 8 (VII/83).- 13 (VIII/85).

var. elongatum Brébisson, en condiciones similares a la especie. Rara.

Localidad: 2 (III/84).

* var. minus Hantzsch, de menor tamaño que el tipo (2100-215 x 12-13 $\mu \mathrm{m})$ y de pared hialina y lisa. En ecología similar al tipo.

Localidad: 3 (III/84).

! Closterium aciculare (Tuffen) West

Aunque es menos frecuente que la especie anterior, aparece también entre los filamentos del plocon (Tabla II).

Localidades: 2 (IV/83).- 3 (IV/83, XII/84).

Closterium dianae Ehrenber (Fig. 1:6)

Forma parte del plocon en charcas someras de las riberas de ríos o ramblas junto con otras especies congéneres, en zonas intensamente iluminadas. (Tabla II).

Localidades: 12 (IV/83).- 11 (V/81).- 8 (VIII/85).- (III/84, IX/85).- 3 (IV/83).- 6 (III/ 84).- 17 (II/85).

Closterium ehrenbergii Meneghini (Fig. 1:5)

Siempre como integrante del plocon, en condiciones diversas de corriente e iluminación (Tabla II). Especie de valor diagnóstico en aguas de corriente intensa, calizas, con pequeñas cantidades de cloruros en ocasiones (Margalef, 1955).

Localidades: 2 (IV/83).- 12 (VI/83).- 18 (VIII/83).- 19 (VII/85).- 20 (VII/83).- 3 (IV/ $83, \mathrm{III} / 84, \mathrm{IX} / 85)$.- $21(\mathrm{~V} / 81)$.

! Closterium gracile Brébisson iluminada.

Entre algas filamentosas de las riberas de cursos de agua alcalina dulce intensamente

Localidad: 3 (IV/83).

Closterium lunula (Müller) Nitzsch (Fig. 1:2)

Plocon de charcas someras próximas a arroyos o de riberas de ríos de escasa corriente y con condiciones de iluminación variables (Tabla II).

Localidades: 12 (IV/83).- 18(VII/83).- 11 (XI/81).-8(VII/83).-2(VI/85).-22 (IV/84).

! Closterium parvulum Nägeli (Fig. 1:4)

Sus células quedan retenidas entre los filamentos de Cladophora en zonas con corrientes, a veces intensa (Tabla II).

Localidades: 3 (IV/83, V/83).- 2 (IX/85).- 8 (VII/83).- 23 (IX/84).- 4 (IX/85). 


\section{! Closterium pronum Brébisson}

Entre los filamentos del plocon en arroyos, en zonas de corriente intensa o lentificada y con insolación variable. (Tabla II).

Localidades: 2 (IV/83).- 3 (IV/83).- 14 (VIII/85).

Closterium ralfsii Brébisson ex Ralfs var. hybridum Rabenhorst

En charcas bastante someras. Conviviendo con otras zigofíceas, en zonas muy iluminadas (Tabla II).

Localidades: 2 (IV/83, V/85)

* Closterium strigosum Brébisson var. elegans (G.S. West) Krieger (Fig. 1:3)

Células hasta veinte veces más largas que anchas $(200 \times 10 \mu \mathrm{m})$. En condiciones variables de corriente entre el plocon de arroyos y ríos (Tabla II).

Localidades: 2 (IV/83).- 11 (V/82).- 20 (VI/83).- 3 (XII/84).

Novedad para la flora española. Citada previamente como C. peracerosum Gay var. elegans G.S. West en Aboal y Llimona (1984).

Closterium turgidum Ehrenberg

Charcas formadas tras los cambios de nivel del río, entre los filamentos de zignematáceas. (Tabla II).

Localidad: $11(\mathrm{~V} / 82)$.

! Pleurotaenium trabecula (Ehrenberg) Nägeli (Fig. 1:7)

Entre los filamentos de Cladophora en condiciones de corriente, a veces muy intensa (Tabla II)

Localidades: 2(IV/83, XII/84, IX/85).- 3 (IV/83, III/84, IX/85).- 8 (VII/83).

* Actinotaenium colpopelta (Brébisson ex Archivald) Compère

Células con forma de guitarra $(48-50 \times 25-27 \mu \mathrm{m})$ y pared celular puntuada. Entre los filamentos del plocon o sobre tapices de cianofíceas en condiciones de corriente intensa. (Tabla II).

Localidades: 2 (XII/84, V/85).- 27 (XI/81).- 28 (IV/82).

Actinotaenium cucurbita (Brébisson) Teiling ex Ruzicka \& Pouzar

En zonas de corriente intensa de arroyos, protegida por los filamentos del plocon. (Tabla II). Típico de superficies higropétricas (Margalef, 1955).

Localidades: 24 (IV/84).- 2 (XII/84).- 3 (III/84).

! Actinotaenium obcuneatum (West) Teiling (Fig. 2:9)

Sobre otras algas, en general cianofíceas, en zonas de corriente intensa. (Tabla II).

Localidades: 1 (III/84).- 27 (XI/81).

* Actinotaenium perminutum (G.S. West) Teiling (Fig. 2:10)

Células muy pequeñas (10-11 x 5-6 $\mu \mathrm{m})$ con un seno poco profundo. Sobre otras algas en zonas de escasa corriente (Tabla II).

Localidad: 4 (VIII/83). 

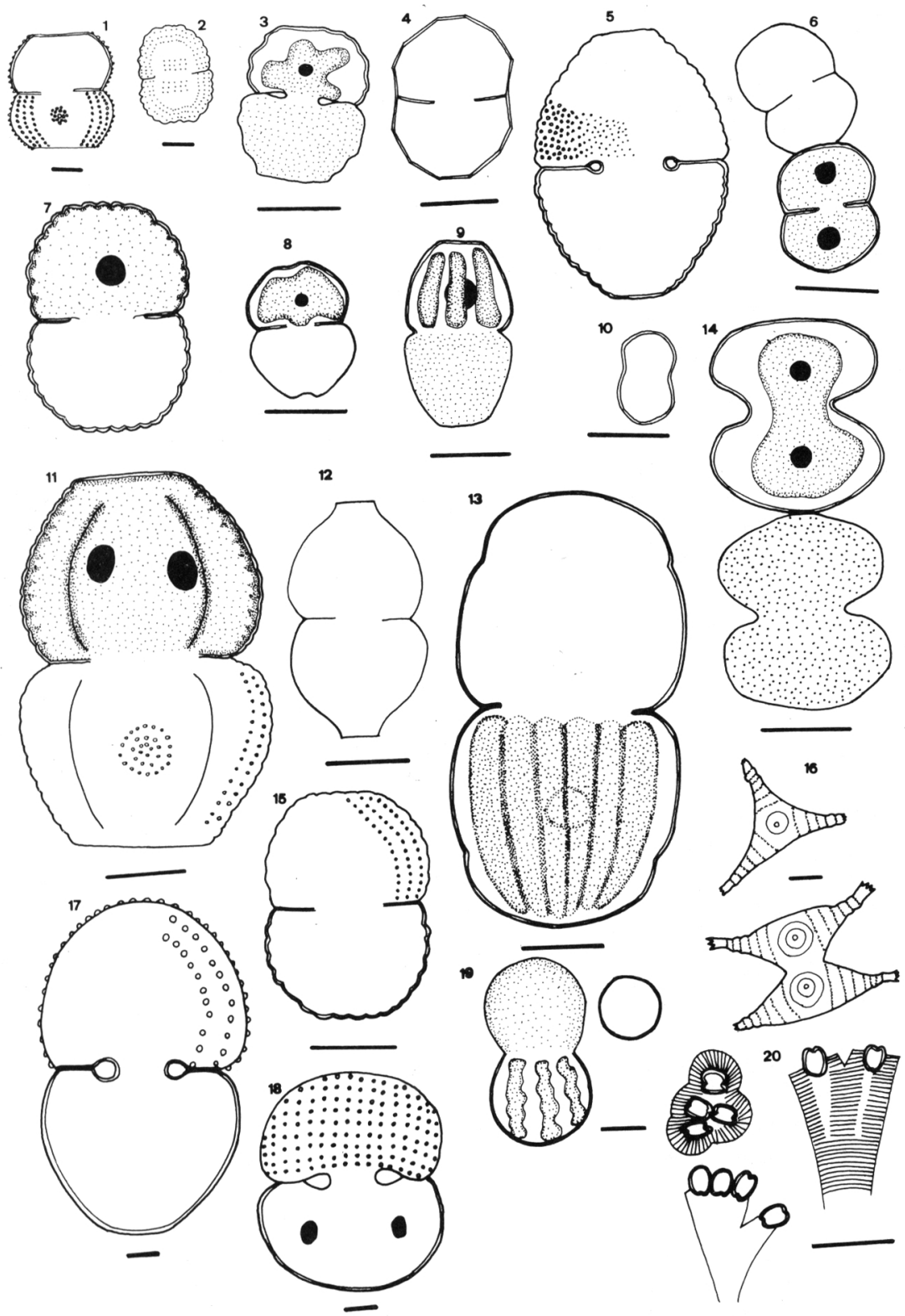

Figura 2.- 1. Cosmarium punctulatum v. subpunctulatum.- 2.C. subcrenatum.- 3.C. venustum.4. C. angulosum.- 5. C. octhodes.- 6. C. wembaerense.- 7. C. undulatum v. minutum.- 8. C. laeve v. westii.- 9. Actinotaenium obcuneatum- 10. A. perminutum.- 11. Staurastrum botrophilum.- 12. Cosmarium granatum f. messikommeri.- 13. C. holmiense.- 14. C. bioculatum.15. C. punctulatum.- 16. Staurastrum paradoxum.- 17. Cosmarium botrytis.- 18. C. reniforme.19. Actinotaenium colpopelta.- 20. Oocardium stratum (La escala representa $10 \mu \mathrm{m}$ ). 
Cosmarium angulosum Brébison (Fig. 2:4)

En pequeñas charcas y entre los filamentos del plocon en riberas de ríos (Tabla III). Localidades: 2 (IV/83_-- 3 (IV/83).- 21 (V/81).

* Cosmarium bioculatum Brébisson (Fig. 2:14)

Células de pequeño tamaño (14-24 x 12-24 $\mu \mathrm{m})$. En charcas someras junto con otras especies congéneres y entre algas filamentosas en las riberas de los ríos. (Tabla III).

Localidades: 3 (IV/83, IX/85).- 2 (IV/83, III/84, IX/85).- 4 (III/84, IX/85).

var. depressum (Schaarschm.) Schmidle

En los mismos ambientes que el tipo.

Localidad: 3 (IV/83).

! Cosmarium botrytis (Meneghini) Ralfs (Fig. 2:17)

Entre las clorofíceas filamentosas del plocon de cursos de agua alcalina dulce. De valor diagnóstico en aguas alcalinas de circulación lenta (Margalef, 1951), muy frecuente en las tierras bajas catalanas (Margalef, 1950).

Localidad: 3 (IV/83)

Cosmarium etchachenense Roy et Bissett

En agua alcalina enriquecida en materia orgánica.

Localidad: 21 (V/82).

Cosmarium garrolense Roy et Bisset

Canal de riego de agua alcalina-dulce, sobre tapices de algas o briófitos, en zonas de corriente relativamente intensa.

Localidad: 29 (XI/81).

* Cosmarium granatum Brébisson ex Ralfs f. messikommeri Croasdale (Fig. 2:12)

Células pequeñas, 1.7 veces más largas que anchas (30-33 x 17-21 $\mu \mathrm{m})$. Entre los filamentos de clorofíceas en arroyos (Tabla III).

Localidades: 2 (III/84, V/85, IX/85).- 4 (VIII/83).- 11 (XI/81).- 3 (III/84).- 30 (I/82).

! Cosmarium holmiense Lundell var. integrum Lundell (Fig. 2:13)

Pequeñas charcas de las riberas del río, entre los filamentos de zignematáceas. Insolación elevada (Tabla III). Característica de superficies higropétricas (Margalef, 1955).

Localidad: 2 (XII/84, V/85).

\section{Cosmarium laeve Rabenhorst}

Forma poblaciones importantes entre los filamentos de clorofíceas de los ríos y arroyos de corriente intensa. Las condiciones de iluminación son variables (Tabla III). Para Margalef (1955) es la especie más común del género y caracteriza las comunidades algales de aljibes de albañilería (Margalef, 1951).

Localidades: 2 (IV/83, III/84).- 3 (IV/83, III/84, IX/85).- 4 (VIII/83).- 11 (IV/82).- 22 (IX/84).- 5 (IX/85).- 6 (XII/84).- 1 (III/84).- 16 (IX/84, II/85, VII/85).- 26 (IV/84).- 27 (XI/ 81).- $31(\mathrm{XI} / 81)$.

var. westii Krieger \& Geroff (Fig. 2:8) 
En ambientes similares al tipo (Tabla III).

Localidades: 1 (III/84).- 25 (IX/84).

! Cosmarium margaritatum (Lundell) Roy et Bisset

Entre otras algas en zonas de corriente relativamente intensa. Iluminación intensa (Tabla III).

Localidades: 2 (IV/83, III/84, XII/84, V/85).- 3 (IV/83).

f. minor (Bold) West et West

De menor tamaño que el tipo $(55 \times 45 \mu \mathrm{m})$. En condiciones similares al tipo.

Localidad: 3 ((IV/83).

! Cosmarium octhodes Nordstedt (Fig. 2:5)

Entre algas filamentosas de las riberas de ríos, intensamente iluminados (Tabla III).

Localidades: 1 (III/84).- 32 (VII/83).- 2 (V/85).

! Cosmarium punctulatum Brébisson (Fig. 2:15)

Forma parte del plocon en riberas de los ríos (Tabla III). Especie tanto de aguas circulantes como estancadas (Margalef, 1955).

Localidades: 1 (III/84).- 2 (IV/83, XII/84).- 3 (IV/83).

var. subpunctulatum (Nordst.) Börgesen (Fig. 2:1)

En ambientes parecidos y conviviendo con el tipo.

Localidad: 2 (III/84).

! Cosmarium reniforme (Ralfs) Archer (Fig. 2:18)

Forma poblaciones relativamente densas, entre las algas filamentosas del plocon de arroyos (Tabla III). Para Margalef (1951) se trata de una especie típica de aguas estancadas eutróficas.

Localidades: 2 (IV/83, III/84, IX/85).- 3 (IV/83, IX/85).- 4 (IX/85).- 5 (IX/85).

! Cosmarium subcrenatum Hantzsch (Fig. 2:2)

Vive junto con otras Desmiciáceas sobre tapices de cianofíceas. Muy iluminada (Tabla III).

Localidades: 1 (III/84).

! Cosmarium tetraophthalmum (Küntzing) Ralfs

Vive en condiciones de corriente e iluminación variables (Tabla III).

Localidades: 2 (V/83, III/84, IX/85).- 4 (VII/83).- 3 (IV/83, III/84, XI/85).- 1 (III/84).

* Cosmarium undulatum Corda ex Ralfs var. minutum Wittrock (Lám. 2:7).

Células de pequeño tamaño, 1.3 veces más largas que anchas (26-28 x 19-21 $\mu \mathrm{m})$. Entre otras algas filamentosas tanto en charcas como en arroyos sometidos a fuerte insolación (Tabla III).

Localidades: 2 (IV/83, III/84, III/85).- 3 (IV/83, III/84).

\section{! Cosmarium vexatum West}

Charcas someras ribereñas (Tabla III). Para Margalef (1951) es una de las especies que caracteriza las comunidades algales de aguas siderotróficas y está ampliamente distribuida 
(Margalef, 1955).

Localidad: 2 (III/84, V/85).

! Cosmarium wembaerense Schmidle (Fig. 2:6)

Entre otras algas en una charca relativamente profunda de agua alcalina dulce, con mineralización elevada y pobre en nutrientes (Tabla III).

Localidad: 16 (II/85).

\section{* Staurastrum botrophilum Wolle (Fig. 2:11)}

Células de mediano tamaño, 1.6-1.8 veces más largas que anchas (45-50 x 25-30 $\mu \mathrm{m}$ ). Convive con numerosas especies de desmidiáceas en pequeñas charcas ribereñas intensamente iluminadas (Tabla III).

Localidad: $2(\mathrm{XII} / 84, \mathrm{~V} / 85)$.

! Staurastrum paradosum Meyen (Fig. 2:16)

Atrapada entre los filamentos de Cladophora en un canal de riego con agua corriente. Localidad: 33 (VIII/85).

\section{Staurastrum punctulatum Brébisson}

En el plocon de balsas de riego o arroyuelos. Características de arroyos de montaña media y alta (Margalef, 1951). Es la especie más común del género (Margalef, 1955).

Localidades: 21 (V/81).- 34 (VIII/85).- 35 (VIII/85).

\section{! Oocardium stratum Nägeli (Fig. 2:19)}

Espífito sobre Batrachospermum moniliforme en ríos de corriente intensa (Tabla III). Característica de superficies, higropétricas (Margalef, 1955).

Localidad: 2 (VI/84).

1, Arroyo de Hondares, Somogil, Murcia, WH 9030. 2, Río Benamor, La Puerta, Moratalla, Murcia, WH 9130. 3, Río Benamor, Cenajo del Agua Cernía, Murcia, WH 8829. 4, Río Benamor, Somogil, Murcia, WH 9030. 5, Río Benamor, Murcia, XH 1034. 6, Río Benamor, Casas de Pelota, Murcia, XH 0030. 7, Río Tus, Fábricas de Madera, Albacete, WH 4044. 8, Río Tus, La Rala, Albacete, WH 5951. 9, Río Madera, Nacimiento, Jaén, WH 3332. 10, Río Madera, Desembocadura, Jaén, WH 3212. 11, Río Mula, Salto Lucero, Bullas, Murcia, XH 1709. 12, Río Bogarra antes de Bogarra, Albacete, WH 6573. 13, Río Segura después del Llano de Molina, Murcia, XH 5414. 14, Río Segura después del embalse de Anchuricas, Jaén, WH 4128. 15, Arroyo Madera, Albacete, WH 5339. 16, Rambla del Puerto, El Portazgo, Murcia, XG 6197. 17, Rambla del Puerto cerca del nacimiento, XG 6294. 18, Arroyo Morote, Albacete, WH 6652. 19, Arroyo Salado, Riópar, Albacete, WH 5260. 20, Arroyo de Haches, Bogarra, Albacete, WH 7375. 21, El Aceniche, Bullas, Murcia, XH 1305. 22, Río de Las Hoyas, Batán del Puerto, Albacete, WH 5670. 23, Río Madera, R. Mundo, Albacete, WH 6172. 24, Río Endrinales en las Espineras, Albacete, WH 5467. 25, Río Endrinales, Albacete, WH 5166. 26 , Río de la Fuente del Roble, Albacete, WH 5873. 27, Caravaca, Canal de riego, Murcia, WH 9916. 28, Carrascalejo, Canal de riego, Bullas, Murcia, XH 1313. 29, Río Argos, Caravaca, Murcia, WH 9916. 30, Río Pliego, Meseta de la Plata, Murcia, XH 3510. 31, Rambla Caputa, Mula, Murcia, XH 3116. 32, Barranco de Ubricas, Murcia, WH 9031. 33, Acequia Barrera, Alcantarilla, Murcia XG 0176. 34, Cañadas de Nerpio, Fuente, Albacete, WH 5315. 35, Cañadas de Nerpio, Balsa, Albacete, WH 5415.

Tabla I. Localidades estudiadas. 
! Hyalotheca dissiliens (Smith) Brébisson ex Ralfs (Fig. 1:10)

En el plocon de riberas de ríos o charcas, junto con otras algas filamentosas, fundamentalmente Zignematáceas (Tabla III).

Localidades: 2 (IV/83).- 4 (IX/85).- 3 (IX/85).- 14 (II/85).- 15 (VII/83).- 10 (VII/83).$16(\mathrm{IV} / 84)$.

AGRADECIMIENTOS. Al Prof. P. Bourrelly (Museum d'Histoire Naturelle. Paris) que nos ayudó con la determinación de algunos táxones y confirmó otros.

\section{BIBLIOGRAFÍA}

ABOAL, M. -1987-Flora algal epicontinental de la Cuenca del río Segura, Murcia. S.E. de España. Tesis Doctoral. Facultad de Biología. Universidad de Murcia.

ABOAL, M. -1988-Zygnemataceae (Conjugales, Chlorophyceae) of the river Segura Basin, Southeastern Spain. Nova Hedwigia, 47(3-4):389-402.

ABOAL, M. y X. LLIMONA - 1984- Aportación al estudio algológico del sistema de Sierras de Ponce y Quípar (NO de Murcia. SE de España). Anales de Biología, 2 (Sección Especial, 2): $1-17$.

KRIEGER, W. -1937-Die Desmidiaceen Europas mit Berüchsichtigung der ausser europaischen Arten. Rabenhorst's Kryptogamen Flora. vol. 13. Leipzig.

KRIEGER, W. \& J. GERLOFF -1962- Die Gattung Cosmarium Lieferung 1. J. Cramer. Weinheim.

KRIEGER, W. \& J. GERLOFF -1965- Die Gattung Cosmarium Lieferung 2. J. Cramer. Weinheim.

KRIEGER, W. \& J. GERLOFF -1969- Die Gattung Cosmarium Lieferung. 3. J. Cramer. Weinheim.

MARGALEF, R. - 1947- Limnosociología. Monografías de Ciencias Modernas nº 10. Instituto Español de Edafología, Ecología y Fisiología Vegetal, nº 4, 93 pp.

MARGALEF, R. -1951- Regiones limnológicas de Cataluña y ensayo de sistematización de algas. Coll. Bot., vol. III, fasc. I, no 2:43-67.

MARGALEF, R. - 1952- Materiales para la hidrobiología de la isla de Menorca. P. Inst. Biol. Apl., XI:5-112.

MARGALEF, R. -1953- Materiales para la hidrobiología de la isla de Mallorca. P. Inst. Biol. Apl., XV:5-111.

MARGALEF, R. -1955- Materiales para una flora de las algas de NE de España. VI. Desmidiales, Rhodophyceae. Coll. Bot., IV, fasc. III, nº 25:319-329.

PRESCOTT, G.W., H.T. CROASDALE, W.C. VINYARD \& C.E. DE M. BICUDO -1981- A synopsis of North American Desmids. Part. II. Desmidiaceae, Placodermae, section 3. University of Nebraska Press.

VIDAL-ABARCA, M.R. -1985- Las aguas superficiales de la Cuenca del Río Segura (S.E. de España). Caracterización físico-química en relación al medio físico y humano. Tesis Doctoral. Facultad de Biología. Universidad de Murcia.

WEST, W. \& G.S. WEST -1904/1923- A monograph of the British Desmidiaceae: I, II, III, IV, V. Ray Society. London.

(Aceptado para su publicación en junio de 1991)

Dirección de la autora: Departamento de Biología Vegetal (Botánica). Facultad de Biología. Campus del Espinardo. Universidad de Murcia. 30071- Murcia. 


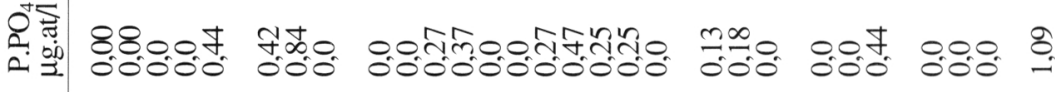

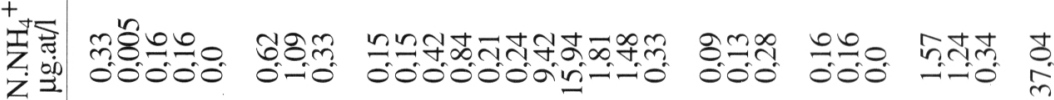

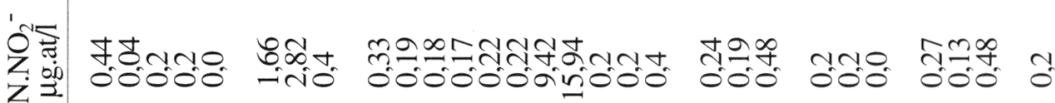

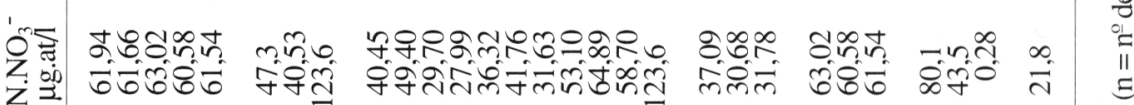

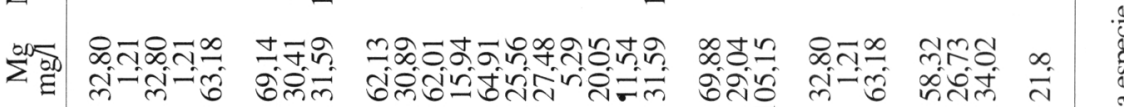

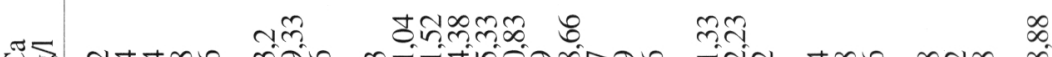

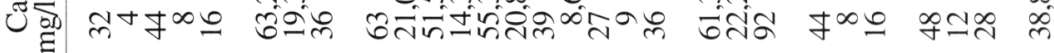

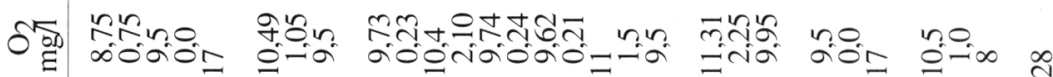

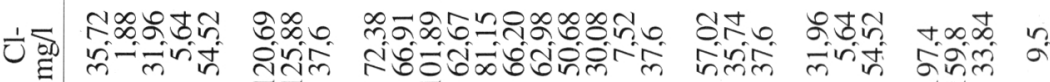

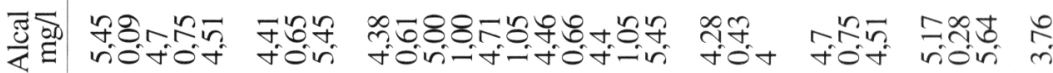

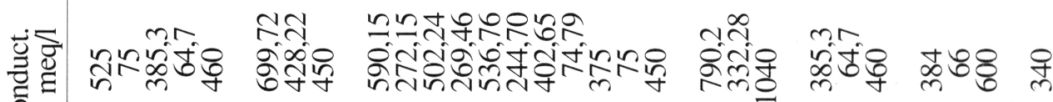

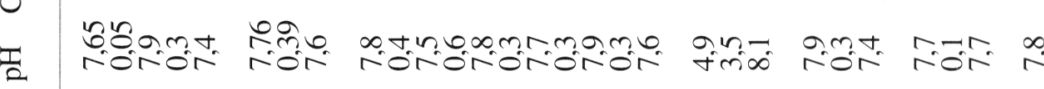

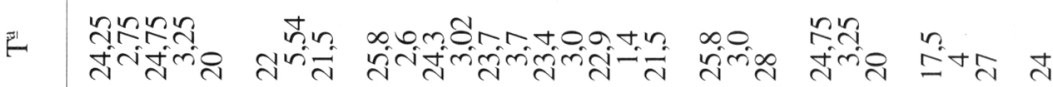

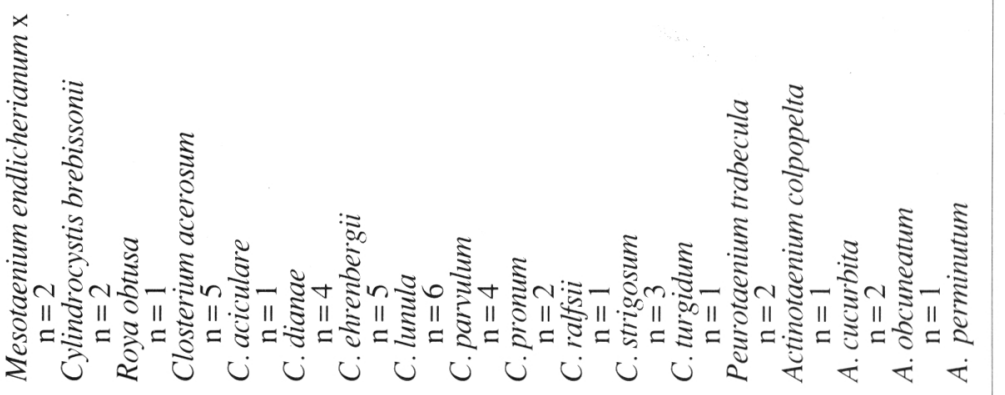




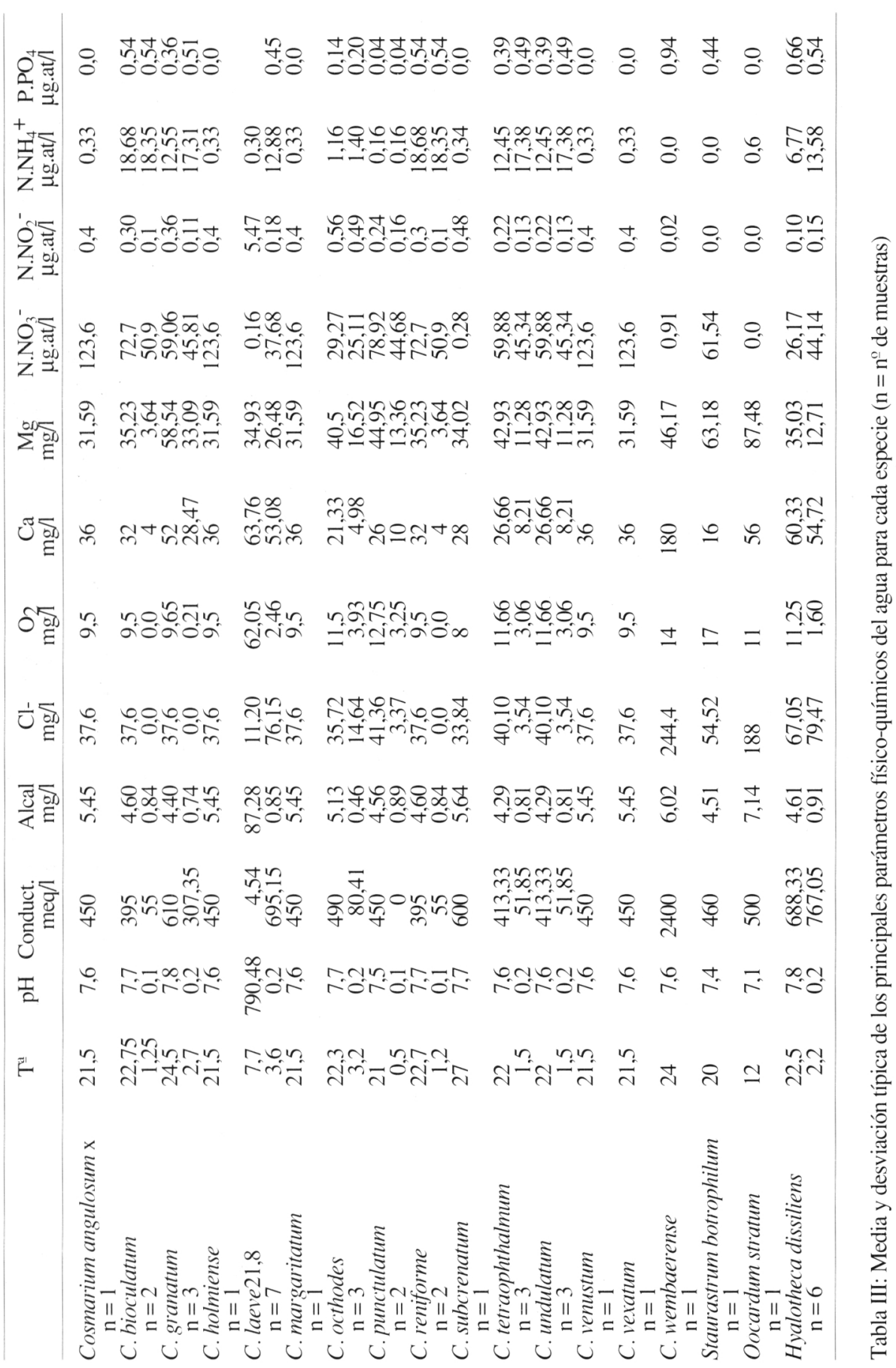

